Ergod. Th. \& Dynam. Sys. (1985), 5, 1-25

Printed in Great Britain

\title{
Finite group actions on shifts of finite type
}

\author{
R. L. ADLER, B. KITCHENS AND B. H. MARCUS
}

IBM T. J. Watson Research Center, P.O. Box 218, Yorktown Heights, NY 10598, USA; IBM Research, 5600 Cottle Road, San Jose, CA 95193, USA and Mathematics Department, University of North Carolina, Chapel Hill, North Carolina 27514, USA

(Received 1 December 1983 and revised 1 October 1984)

Abstract. A continuous $\mathbb{Z} \otimes_{T} G$ action on a subshift of finite type consists of a subshift of finite type with its shift transformation, together with a group, $G$, of homeomorphisms of the subshift and a group automorphism $T$, so that the commutation relation $\sigma \circ g=T g \circ \sigma$ is satisfied, where $\sigma$ is the shift and $g$ is any element of $G$.

Here, we investigate these actions when $G$ is finite. We show that if $\Sigma_{A}$ is any positive entropy subshift of finite type, $G$ is any finite group and $T$ is any automorphism of $G$ then there is a non-trivial $\mathbb{Z} \otimes_{T} G$ action on $\Sigma_{A}$. We then classify all such actions up to 'almost topological' conjugacy.

\section{Introduction}

A continuous $\mathbb{Z} \otimes_{T} G$ action on a subshift of finite type (ssft), $\Sigma_{A}$, consists of a group, $G$, of homeomorphisms of $\Sigma_{A}$ and an automorphism $T$ of $G$ satisfying $\sigma \circ g=T g \circ \sigma$ where $\sigma$ is the shift. The problem we consider is that of classifying these actions up to almost topological conjugacy. When $G$ is a finite group we give necessary and sufficient conditions for this classification. This type of group action arises naturally and is crucial in classifying equal entropy factors and extensions of ssft. We will treat this topic in a subsequent paper. The parallel measure-theoretic problems have been investigated by $D$. Rudolph in [6] and [7]. We obtain the same conditions as Rudolph but in our context the proofs are quite different.

The overall approach to proving the main theorem is to follow the line of reasoning in [1] as closely as possible. There it is shown that if two irreducible ssft have the same entropy and ergodic period they are almost topologically conjugate. Knowledge of this argument is not taken for granted, it is contained in this discussion in a slightly altered form. However, a certain familiarity with ideas and constructions common to symbolic dynamics is assumed. All proofs (except in the last section) are complete and self-contained but the reader unfamiliar with this type of argument may find them somewhat sketchy.

We begin in $\S 1$ with a little background material. The necessary notation and definitions are set up. $\S 2$ examines groups acting on ssft. There are a number of observations made about these actions when the group is of the form $\mathbb{Z} \otimes_{T} G$, with $G$ finite. We also observe that if $G$ is any finite group, $T$ is any automorphism and $\Sigma_{A}$ is any ssft then there is a $\mathbb{Z} \otimes_{T} G$ action on $\Sigma_{A}$. Next we examine the case where $G$ is a compact topological group and there is a $\mathbb{Z} \otimes_{T} G$ action on an ssft. This 
implies that $G$ must be zero-dimensional and we show that if $T^{p}=$ identity for some $p \neq 0$ we may regard it as a finite group. After this we prove that if $G$ is finite and there is a $\mathbb{Z} \otimes_{T} G$ action on an ssft, we can find an extension of this action where the new $\mathbb{Z} \otimes_{T} G$ action has a simple form that allows us to simplify many of the constructions that follow.

$\S 3$ proceeds along the lines of the argument in [1]. The steps are the same and it is shown that each of the constructions made there can be repeated here if we are sufficiently careful. We prove that if $\Sigma_{A}$ and $\Sigma_{B}$ are aperiodic ssft with the same entropy, and both have $\mathbb{Z} \otimes_{T} G$ actions then they are almost topologically conjugate.

$\S 4$ deals with irreducible but periodic ssft. When two ssft have $\mathbb{Z} \otimes_{T} G$ actions, the same entropy and ergodic period greater than one, we see that another obstruction to almost topological conjugacy may exist. The subgroup that fixes each cyclic subset, as well as certain properties of its cosets must be taken into account. We show that once this extra condition is met the two $\mathbb{Z} \otimes_{T} G$ actions will be almost topologically conjugate.

Finally, in the last section we observe that all these results carry over to sofic systems.

The authors would like to thank Dan Rudolph for his discussions on these topics.

R. L. Adler was partially supported by NSF Grant MCS-81-07092. B. H. Marcus was partially supported by NSF Grant MCS-83-01246.

\section{Background}

In the discussion that follows we will assume the reader is familiar with the basic ideas concerning subshifts of finite type ( $\mathrm{ssft}$ ). These include irreducibility, aperiodicity, Perron-Frobenius theory, topological entropy, ergodic period, block maps, cylinder sets, Markov partitions, and the basic operations of going to higher blocks, splitting states, and amalgamating states. All of these ideas are discussed in [1], and the basic operations are also examined in $\S 2$.

For a zero-one transition matrix, $A$, which we will always assume to be irreducible with spectral radius greater than one, $\Sigma_{A}$ will denote the ssft defined by $A, L_{A}$ will denote the alphabet of $\Sigma_{A}$ and $\lambda_{A}$ (or $\lambda$ ) will denote $A$ 's maximal eigenvalue. For a symbol $I \in L_{A}$ let

(or $f(I)$ ) and

$$
f_{A}(I)=\left\{J \in L_{A}: A_{I J}=1\right\}
$$

$$
p_{A}(I)=\left\{K \in L_{A}: A_{K I}=1\right\} .
$$

These are the successor and predecessor sets of a symbol. Specifically, they will be denoted by

$$
f(I)=\left\{I_{1}, \ldots, I_{|f(I)|}\right\} \text { and } p(I)=\left\{I_{1}^{*}, \ldots, I_{|p(I)|}^{*}\right\} .
$$

For a subset $E \subseteq L_{A}$ let

$$
f(E)=\bigcup_{I \in E} f(I) \quad \text { and } \quad p(E)=\bigcup_{I \in E} p(I) .
$$

If $\phi: \Sigma_{A} \rightarrow \Sigma_{B}$ is a one-block map then we will use $\phi$ interchangeably to stand for the map from $\Sigma_{A}$ to $\Sigma_{B}$ or the map from $L_{A}$ to $L_{B}$. If $\phi: \Sigma_{A} \rightarrow \Sigma_{B}$ is a continuous, 
onto, shift-commuting map then we will say that $\Sigma_{A}$ is an extension of $\Sigma_{B}$ and $\Sigma_{B}$ is a factor of $\Sigma_{A}$ or that $\phi$ is a factor map. Right or left-resolving is a property of some factor maps that we will use repeatedly. Say a map $\phi: \Sigma_{A} \rightarrow \Sigma_{B}$ is right resolving if it is a one-block map and $\phi: f_{A}(I) \rightarrow f_{B}(\phi(I))$ defines a set isomorphism for each $I \in L_{A}$. Similarly, say $\phi: \Sigma_{A} \rightarrow \Sigma_{B}$ is left resolving if it is a one-block map and $\phi: p_{A}(I) \rightarrow p_{B}(\phi(I))$ defines a set isomorphism for each $I \in L_{A}$. A right (or left) resolving map is always onto and boundedly finite-to-one (see [1] for a discussion).

A factor map $\phi: \Sigma_{A} \rightarrow \Sigma_{B}$ is said to be one-to-one a.e. if it is one-to-one when restricted to the doubly transitive points of $\Sigma_{A}$ and $\Sigma_{B}$. In this case $\Sigma_{A}$ is said to be an almost conjugate extension of $\Sigma_{B}$ and $\Sigma_{B}$ is an almost conjugate factor of $\Sigma_{A}$. This is not the definition in [1] but the two definitions are equivalent when dealing with subshifts of finite type. A discussion of some of this can be found in [2]. A map $\phi: \Sigma_{A} \rightarrow \Sigma_{B}$ is one-to-one a.e. if and only if a resolving block (or magic-word) exists for $\phi$. A resolving block (or magic-word) for $\phi$, when it is recoded to a one block map, is a cylinder set $\left[J_{1}, \ldots, J_{n}\right]$ in $\Sigma_{B}$ whose pre-image

$$
\phi^{-1}\left(\left[J_{1}, \ldots, J_{n}\right]\right)=\bigcup_{k=1}^{\imath}\left[I_{1}^{k}, \ldots, I_{n}^{k}\right]
$$

is composed of blocks that all agree in some entry, $I_{m}^{k}=I$ for some $m$ and all $k$. Two ssft $\Sigma_{A}$ and $\Sigma_{B}$ are said to be almost topologically conjugate when there exists a common almost conjugate extension.

\section{Group actions and skew group actions}

Let $\Sigma_{A}$ be an ssft, $G$ be a topological group, $T: G \rightarrow G$ an automorphism. We say $G$ acts (continuously) on $\Sigma_{A}$ if

(i) each $g \in G$ defines a homeomorphism $g: \Sigma_{A} \rightarrow \Sigma_{A}$, so that the map $(g, x) \rightarrow g(x)$ is also continuous;

(ii) $g(h(x))=g h(x)$, i.e. group multiplication is the same as map composition;

(iii) for each $g \in G, g \neq$ id there is an $x$ (in each cyclic subset, if $\Sigma_{A}$ is periodic) so that $g(x) \neq x$.

If $\sigma \circ g=g \circ \sigma$ for each $g \in G$ we say we have a $\mathbb{Z} \times G$ action on $\Sigma_{A}$. If $\sigma \circ g=T g \circ \sigma$ we say we have a $\mathbb{Z} \otimes_{T} G$ action or $G$ skew action on $\Sigma_{A}$.

Example 1. Let $S(2)=\{(1,2),(2,1)\}$ be the permutation group on two elements. Define a $\mathbb{Z} \times S(2)$ action on the full two-shift, $\{1,2\}^{\mathbb{Z}}$, by letting $S(2)$ act on $L_{A}=\{1,2\}$ in the natural way.

Example 2. Define $\Sigma_{A}$ by

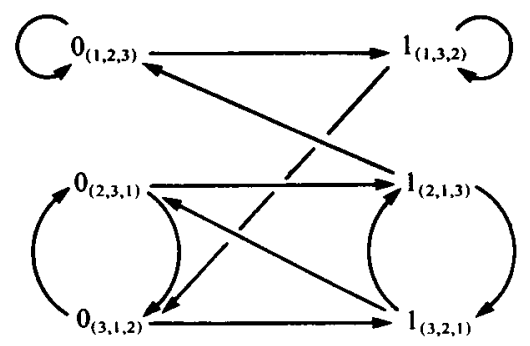


Let $S(3)=\{(1,2,3),(1,3,2),(2,1,3),(2,3,1),(3,1,2),(3,2,1)\}$ be the permutation group on three elements. Let $G=\{(1,2,3),(2,3,1),(3,1,2)\}$ be a subgroup of $S(3)$. Let $T: G \rightarrow G$ be defined by

$$
T(i, j, k)=(1,3,2)(i, j, k)(1,3,2) .
$$

Define a $\mathbb{Z} \otimes_{T} G$ action on $\Sigma_{A}$ by letting $G$ act on $L_{A}$ by permuting the subscripts in the natural way, $g\left(I_{h}\right)=I_{g h}$.

A factor map from a $\mathbb{Z} \otimes_{T} G$ action on $\Sigma_{A}$ to a $\mathbb{Z} \otimes_{T} G$ action on $\Sigma_{B}$ is a factor map between $\Sigma_{A}$ and $\Sigma_{B}$ that also commutes with each element of $G$. In this case we say that the $\mathbb{Z} \otimes_{T} G$ action on $\Sigma_{B}$ is a factor of the one on $\Sigma_{A}$, and that the action on $\Sigma_{A}$ is an extension of the one on $\Sigma_{B}$. If the map is a homeomorphism we say the actions are topologically conjugate. Two $\mathbb{Z} \otimes_{T} G$ actions are almost topologically conjugate if they have a common one-to-one a.e. extension. When $G$ is a finite group we make the following observations.

Observation 1 . Every $\mathbb{Z} \otimes_{T} G$ action is topologically conjugate to a one-block action, i.e. there is a $G$ action on $L_{A}$ so that the $G$ part of the $\mathbb{Z} \otimes_{T} G$ action acts on $\Sigma_{A}$ by

$$
g(x)=\left(\ldots, T^{-1} g\left(x_{-1}\right), g\left(x_{0}\right), T g\left(x_{1}\right), \ldots\right) .
$$

Observation 2. If we have a one-block $\mathbb{Z} \otimes_{T} G$ action on $\Sigma_{A}$ and $P_{g}$ is the permutation matrix indexed by $L_{A}$ that represents $g$, then $P_{g} A=A P_{T_{g}}$ for all $g \in G$. This means $p(g(I))=T^{-1} g(p(I))$ and $f(g(I))=T g(f(I))$ for all $I \in L_{A}$ and $g \in G$.

Observation 3. If we have a $\mathbb{Z} \otimes_{T} G$ action on $\Sigma_{A}$ then $g(x) \neq x$ for all $g \in G$ and all doubly transitive $x \in \Sigma_{A}$.

Proofs. (1) Let $\mathscr{P}$ be the partition on $\Sigma_{A}$ of one-block time zero cylinder sets. Define a new partition, $\mathscr{P}^{\prime}=\bigvee_{g \in G} g(\mathscr{P})$. This new partition is finite, invariant under $G$, and Markov with respect to $\sigma$. Let $\boldsymbol{A}^{\prime}$ be the transition matrix for this new partition. Then $\Sigma_{A^{\prime}}$ is a new ssft, topologically conjugate to $\Sigma_{A}$, with a one-block $\mathbb{Z} \otimes_{T} G$ action.

(2) These three equations follow directly from the fact that $\mathbb{Z} \otimes_{T} G$ is a one-block action, using $\sigma \circ g=T g \circ \sigma$.

(3) Since $T$ is an automorphism of a finite group there is a $p$ such that $T^{p}=\mathrm{id}$. Then the $\mathbb{Z} \otimes_{T} G$ action on $\Sigma_{A}$ gives a $\mathbb{Z} \times G$ action on each irreducible component of $\Sigma_{A^{p}}$. Consider each one of these separately. Observe that if $x$ is doubly transitive for $\sigma$ on $\Sigma_{A}$, then $x$ is doubly transitive on its component for $\sigma^{p}$. So we are reduced to the case of a one-block $\mathbb{Z} \times G$ action on an irreducible ssft. For each $g \in G$ there is an $I \in L_{A}$ such that $g(I) \neq I$; since every doubly transitive point, $x$, contains $I$, $g(x) \neq x$.

At this point we will digress to examine how a skew group action affects the standard higher block, state splitting, and amalgamation operations.

Higher blocks. Consider a $\mathbb{Z} \otimes_{T} G$ action on an ssft $\Sigma_{A}$. Define a natural conjugate action on the two-block system of $\Sigma_{A}$ by:

$$
g([I, J])=[g(I), \operatorname{Tg}(J)], \quad \forall g \in G .
$$


This gives a one-block $\mathbb{Z} \otimes_{T} G$ action on the two block system because if $[I, J] \rightarrow[J, K]$,

$$
g([I, J])=[g(I), T g(J)] \rightarrow\left[T g(J), T^{2} g(K)\right]=T g[J, K]
$$

The construction extends to the $k$-block system in the natural way.

State splitting. Fix an $I \in L_{A}$ and let $f(I)=E_{1} \cup E_{2}$ be a partition of the follower set of $I$ into two sets. When $I \notin f(I)$ split $I$ into two symbols $I^{1}, I^{2}$ with

$$
p\left(I^{1}\right)=p\left(I^{2}\right)=p(I), \quad f\left(I^{1}\right)=E_{1} \quad \text { and } \quad f\left(I^{2}\right)=E_{2} .
$$

When $I \in f(I)$ suppose $I \in E_{1}$, split $I$ into two symbols $I^{1}, I^{2}$ with $p\left(I^{1}\right)=p\left(I^{2}\right)=(p(I)-\{I\}) \cup\left\{I^{1}\right\}, \quad f\left(I^{1}\right)=\left(E_{1}-\{I\}\right) \cup\left\{I^{1}, I^{2}\right\} \quad$ and $f\left(I^{2}\right)=E_{2}$.

This defines a new ssft conjugate to the original. If we also want to get a conjugate $\mathbb{Z} \otimes_{T} G$ action, then we must split more states. For each $g \in G$, split each state $g(I)$ according to the partition $f(g(I))=\operatorname{Tg}\left(E_{1}\right) \cup \operatorname{Tg}\left(E_{2}\right)$. This splits each $g(I)$ into $(g(I))^{1}$ and $(g(I))^{2}$ as before. For $h \in G$ define $h\left((g(I))^{1}\right)=(h g(I))^{1}$ and $h\left((g(I))^{2}\right)=(h g(I))^{2}$. This defines a new one-block $\mathbb{Z} \otimes_{T} G$ action. The construction may be carried out using the predecessors instead of the followers.

Amalgamations. Let $I, J \in L_{A}$ with $I \neq J, p(I)=p(J)$ and $f(I) \cap f(J)=\varnothing$. Then the amalgamation $I \sim J$, defines an ssft conjugate to $\Sigma_{A}$; namely, throw out the states $I$ and $J$, and add the state $\{I, J\}$ with transitions

$$
p(\{I, J\})=p(I)=p(J), \quad f(\{I, J\})=f(I) \cup f(J),
$$

if $I$ or $J$ is not in $f(I) \cup f(J)$. If $I$ or $J$ is in $f(I) \cup f(J)$ we may without loss of generality assume $\{I, J\} \cap(f(I) \cup f(J))=\{I\}$. This means $I \in p(I)=p(J)$. Now the amalgamation $I \sim J$ defines an ssft conjugate to $\Sigma_{A}$ with

$$
\begin{aligned}
& p(\{I, J\})=(p(I)-\{I\}) \cup\{\{I, J\}\}, \\
& f(\{I, J\})=(f(I)-\{I\}) \cup f(J) \cup\{\{I, J\}\} .
\end{aligned}
$$

In order to get an amalgamation that respects the $\mathbb{Z} \otimes_{T} G$ action, we assume $J \notin G(I)=\{g(I): g \in G\}$ and then amalgamate the other pairs $g(I) \sim g(J), g \in G$. This all fits together since

$$
p(g(I))=T^{-1} g(p(I))=T^{-1} g(p(J))=p(g(J)),
$$

and

$$
f(g(I)) \cap f(g(J))=T g(f(I)) \cap T g(f(J))=\varnothing .
$$

A $G$-action is defined by

$$
h(\{g(I), g(J)\})=\{h g(I), h g(J)\} .
$$

This gives a $\mathbb{Z} \otimes_{T} G$ action on the new ssft that is naturally conjugate to the original. This procedure may also be carried out if the roles of predecessors and successors are exchanged.

Next, we observe that $\mathbb{Z} \otimes_{T} G$ actions, for finite $G$, are plentiful.

THEOREM 1. If $G$ is any finite group, $T: G \rightarrow G$ is any automorphism, $\Sigma_{A}$ is any irreducible ssft then there is a $\mathbb{Z} \otimes_{T} G$ action on $\Sigma_{A}$. 
Proof. This is essentially the proof of the corresponding statement in [3]. Let $G, T$, and $\Sigma_{A}$ be as in the hypothesis. First we will produce a $\mathbb{Z} \times G$ action on $\Sigma_{A}$. Since every finite group is isomorphic to a subgroup of the permutation group $S(n)$ for sufficiently large $n$, it suffices to produce a $\mathbb{Z} \times S(n)$ action. Choose a simple cycle, $\left[I_{1}, I_{2}, \ldots, I_{p}, I_{1}\right]$, in the graph of $A$. By using the elementary conjugacies (of course without the group action) just discussed, if necessary, we can assume there is a $J \in f\left(I_{1}\right), J \neq I_{2}$ and a $K \in p\left(I_{1}\right), K \neq I_{p}$. Choose an integer $N$ large enough so that $\left(A^{N-1}\right)_{J K}>n$. Choose $n$ of these $N$-blocks and number them. $S(n)$ can act on these blocks by permutation. Define maps on $\Sigma_{A}$ by letting $g \in S(n)$ act on $x$ by leaving

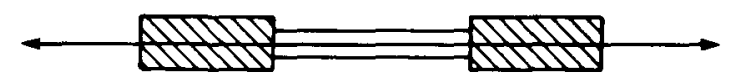

all blocks in $x$ alone except when one of the numbered blocks occurs between two blocks of $\left[I_{1}, \ldots, I_{p}, I_{1}\right]$ repeated cyclically of length at least $2 N$. When such a configuration occurs, the numbered block is permuted by $g$. The cyclic blocks of length at least $2 N$ are markers needed to insure that the numbered blocks being permuted don't interfere with one another. This gives a $\mathbb{Z} \times G$ action for any finite $G$. Now we easily produce the $\mathbb{Z} \otimes_{T} G$ action. Permute the blocks by $g$ if the numbered block begins at time zero, $x_{0}=J$. If the numbered block begins at time $n, x_{n}=J$, permute it by $T^{n} g$.

Next we examine the case where the group acting is a compact topological group. We conclude that in many cases, because of the topology of $\Sigma_{A}$, there are really no new actions.

Observation 4. If $G$ is a compact topological group and we have a $\mathbb{Z} \otimes_{T} G$ action on $\Sigma_{A}$, then $G$ is zero-dimensional.

Proof. First notice that if $x \in \Sigma_{A}$ is doubly transitive then $g(x) \neq x$ for all $g \neq$ identity. Fix a doubly transitive $x \in \Sigma_{A}$, so $G(x) \subseteq \Sigma_{A}$ is a closed subset of $\Sigma_{A}$. Define a map from $G(x)$ to $G$ by sending $y \in G(x)$ to the unique element $g \in G$ where $g(x)=y$. This is a homeomorphism which also defines a group operation on $G(x)$ by

$$
y \circ z=g(x) \circ h(x)=g h(x)=w .
$$

This makes the map a group isomorphism, $G(x) \cong G$.

Example 3. Let $\Sigma_{A}$ be the full four-shift with alphabet $L_{A}=\{(0,0),(0,1)$, $(1,0),(1,1)\}$. Let $G=\{0,1\}^{\mathbb{Z}}$ with coordinate wise addition modulo two the group operation. Let $T$ be the shift. Define a $\mathbb{Z} \otimes_{T} G$ action on $\Sigma_{A}$ by

$$
g(x)=\cdots\left(x_{-1}^{1}, x_{-1}^{2}+g_{-1}\right)\left(x_{0}^{1}, x_{0}^{2}+g_{0}\right)\left(x_{1}^{1}, x_{1}^{2}+g_{1}\right) \cdots
$$

where

$$
\begin{aligned}
& x=\cdots\left(x_{-1}^{1}, x_{-1}^{2}\right)\left(x_{0}^{i}, x_{0}^{2}\right)\left(x_{1}^{1}, x_{1}^{2}\right) \cdots \\
& g=\cdots g_{-1} g_{0} g_{1} \cdots
\end{aligned}
$$

and the additions are modulo two.

We would like to thank D. Lind for his help with the preceding observation and example. 
THEOREM 2. Assume $G$ is a compact topological group with an automorphism $T$, $T^{p}=$ identity for some $p$, and we have a $\mathbb{Z} \otimes_{T} G$ action on $\Sigma_{A}$. Then there is a finite group $G^{\prime}$ with an automorphism $T^{\prime}$, and a group homomorphism $\rho: G \rightarrow G^{\prime}$ giving rise to $a \mathbb{Z} \otimes_{T^{\prime}} G^{\prime}$ action, such that $\rho \circ T=T^{\prime} \circ \rho$ and $g=\rho(g)$ as a map of $\Sigma_{A}$, for each $g \in G$.

Proof. Assume $G$ has a one-block action on $\Sigma_{A}$. Define an equivalence relation on $G$ by saying $g \sim_{0} h$ if and only if $g(I)=h(I)$ for all $I \in L_{A}$. The partition $\mathscr{P}_{0}$ of $G$ defined by this equivalence relation is finite and open-closed whenever $G$ acts continuously on $\Sigma_{A}$. Define a second equivalence relation on $G$ by saying $g \sim h$ if and only if $g(x)=h(x)$ for all $x \in \Sigma_{A}$. Both equivalence relations are preserved by the group operation. This means the quotient topological group defined by second relation $G / \sim=G^{\prime}$ acts continuously on $\Sigma_{A}$. The quotient map $\rho: G \rightarrow G^{\prime}$ makes $g=\rho(g)$ as homeomorphisms of $\Sigma_{A}$ and also induces an automorphism $T^{\prime}$ of $G^{\prime}$. The partition of $G$ defined by the second equivalence relation is $\mathscr{P}=\bigvee_{i=-\infty}^{\infty} T^{i}\left(\mathscr{P}_{0}\right)$. Any time this is finite $G^{\prime}$ is finite. We have assumed $T^{P}$ is the identity for some $p$, so in this case it is finite.

This raises the question of what are necessary and sufficient conditions on $G$ and $T$ to have a $\mathbb{Z} \otimes_{T} G$ action on a given $\Sigma_{A}$.

Suppose we have a $\mathbb{Z} \otimes_{T} G$ action on $\Sigma_{A}$, for $G$ finite. If each $g \in G$ is a one-block map, and for $g \neq$ identity, $g(I) \neq I$ all $I \in L_{A}$, say we have a special $\mathbb{Z} \otimes_{T} G$ action on $\Sigma_{A}$. The following theorem allows us to deal only with special actions in the rest of this paper. This will greatly simplify the main constructions.

THEOREM 3. If we have a $\mathbb{Z} \otimes_{T} \dot{G}$ action on $\Sigma_{A}$, for finite $G$ and irreducible $\Sigma_{A}$, then there is a $\Sigma_{\bar{A}}$ with a special $\mathbb{Z} \otimes_{T} G$ action that is an almost conjugate extension of the $\mathbb{Z} \otimes_{T} G$ action on $\Sigma_{A}$. The factor map can be made to be either left or right resolving.

Proof. We will first examine the case where we have a $\mathbb{Z} \times G$ action (see example 4). The construction we make is simpler when there is no skewing function. Assume that we have recoded so that we are dealing with a one-block $G$ action, and notice that if $g(x) \neq x$ for all $g \neq$ identity and $x \in \Sigma_{A}$ then we need only go to a higher block presentation to arrive at the desired result. Suppose this is not the case. We shall show that by going to a sufficiently high block system we can choose a $K$ in the alphabet so that:

(i) $g(K) \neq K$ for each $g \in G, g \neq$ identity;

(ii) the ssft $\Sigma_{F}$ obtained by deleting the set $G(K)$ from the alphabet of $\Sigma_{A}$ is irreducible and has the same ergodic period as $\Sigma_{A}$.

To see that this is possible, first notice that it is possible to choose a block $B$ in $\Sigma_{A}$ so that $g(B) \neq B$ for each $g \in G, g \neq$ identity. Fix this block and let $I$ be the first symbol of $B$ and $J$ the last. Make sure $B$ is long enough so that there is a block $B^{\prime}$ in $\Sigma_{A}$ that has the same length as $B$, begins with $I$, ends with $J$, and is not in $G(B)$. Next find two blocks $M$ and $N$ so that the block $M B N$ is in $\Sigma_{A}$ and so that $M B N$ cannot overlap itself in such a way that the $B$ 's overlap. This is choosing markers just as in the proof that all $\mathbb{Z} \otimes_{T} G$ actions exist for $G$ finite. The construction there will produce such an $M$ and $N$. 


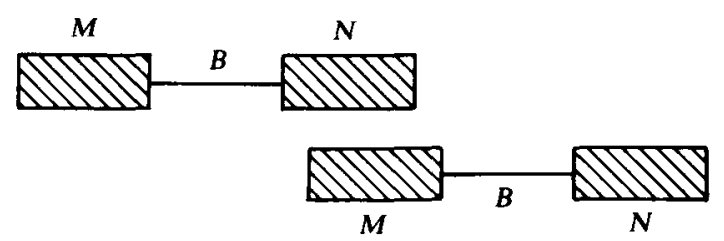

Do this for all blocks in $G(B)$ so that no block gi $(M B N)$ can overlap $M B N$ so much that $g(B)$ and $B$ overlap. Let $\Sigma_{F}$ be the ssft obtained by deleting $G(M B N)$ from the blocks of $\Sigma_{A} . \Sigma_{F}$ is irreducible. To prove this we will produce a doubly transitive point for $\Sigma_{F}$. Take any doubly transitive point $x \in \Sigma_{A}$. Let $y$ be the point in $\Sigma_{F}$ obtained by replacing each occurrence of $g(M B N)$ in $x$ by the block $g(M) g\left(B^{\prime}\right) g(N)$ for each $g \in G$. This is a doubly transitive point for $\Sigma_{F}$. To see that $\Sigma_{F}$ can be made to have the same period we need only to choose $x$ and $y$ periodic in $\Sigma_{A}$ with $\operatorname{gcd}\{$ period $x$, period $y\}=$ period of $\Sigma_{A}$ and then choose $B$ so that $x$, $y \in \Sigma_{F}$. Now go to a sufficiently high block presentation so that $K=M B N$ is in the alphabet.

Once $K$ is chosen and $\Sigma_{F}$ is produced the rest is easy. The idea is to make an extension of $\Sigma_{A}$ so that $\Sigma_{F}$ has exactly $|G|$ pre-images and the symbol $K$ has one. Assume now we have $L_{F} \subseteq L_{A}, G(K)=L_{A}-L_{F}$, and $G$ acts as one-block maps on $L_{A}$. Define $\Sigma_{\bar{A}}$ by

$$
L_{\bar{A}}=\left(L_{A}-L_{F}\right) \cup\left(L_{F} \times G\right)
$$

and say

$$
\begin{aligned}
I \rightarrow J & \text { if } I \rightarrow J \text { in } \Sigma_{A}, \\
I \rightarrow(J, g) & \text { if } I \rightarrow J \text { in } \Sigma_{A}, \\
(I, g) \rightarrow(J, g) & \text { if } I \rightarrow J \text { in } \Sigma_{A}, \\
(I, g) \rightarrow J & \text { if } J=g(K) \text { and } I \rightarrow J \text { in } \Sigma_{A} .
\end{aligned}
$$

This is irreducible because $\Sigma_{A}$ and $\Sigma_{F}$ are. Define a $\mathbb{Z} \times G$ action on $\Sigma_{\bar{A}}$ by:

$$
h(I) \text { is defined as on } L_{A}, h(I, g)=(h(I), h g) \text {. }
$$

Notice that this is a special action since $(I, g) \neq(h(I), h g)$ unless $h=$ identity. The obvious map $\Sigma_{\bar{A}} \rightarrow \Sigma_{A}$ is left resolving, one-to-one a.e., $|G|$-to-one at most, and commutes with the $\mathbb{Z} \times G$ actions. Notice that every element of $G(K)$ is a magic word and that the map is exactly $|G|$-to-one on $\Sigma_{F}$. The map could have been made right resolving instead of left by reversing the roles of the successors and predecessors in the construction of $\Sigma_{\bar{A}}$.

The construction for a $\mathbb{Z} \otimes_{T} G$ action uses the same idea but is slightly more involved. The idea is instead of covering $\Sigma_{F}$ by $|G|$ copies of itself we cover it with $|G|$ copies of a pertiodic ssft. The standard $p$ periodic cover (or extension) of an ssft type is defined as follows. Let $L=L_{F} \times\{0, \ldots, p-1\}$. Let $q$ be the period of $\Sigma_{F}$ and $L_{F}^{0}, \ldots, L_{F}^{q-1}$ be the decomposition of $L_{F}$ into its cyclic subsets. Then say $(I, k) \rightarrow(J, l)$ if and only if $I \rightarrow J$ in $\Sigma_{F}$ and either (i) $l=k+1 \leq q-1$, or (ii) $k=q-1$ and $l=0$. This new ssft is irreducible and has period $p$ times the period of $\Sigma_{F}$. The obvious map from it onto $\Sigma_{F}$ is exactly $p$-to-one. The covers of $\Sigma_{F}$ in the new 
construction won't be copies of $\Sigma_{F}$ now, but will be standard $p$ periodic covers of $\Sigma_{F}$, where $p$ is the period of $T$.

To begin the construction assume we have a $\mathbb{Z} \otimes_{T} G$ action on an irreducible ssft $\Sigma_{A}$. Choose $\Sigma_{F}$ as before and let $p$ be the period of $T$. Define $\Sigma_{\bar{A}}$ by

and say

$$
L_{\bar{A}}=\left(L_{A}-L_{F}\right) \cup\left(L_{F} \times G \times\{0, \ldots, p-1\}\right),
$$

$$
\begin{aligned}
I \rightarrow J & \text { if } I \rightarrow J \text { in } \Sigma_{A}, \\
I \rightarrow(J, g, n) & \text { if } I \rightarrow J \text { in } \Sigma_{A}, \\
(I, g, n) \rightarrow(J, g, n+1) \bmod p, & \text { if } I \rightarrow J \text { in } \Sigma_{A}, \\
(I, g, 0) \rightarrow J & \text { if } J=g(K) \text { and } I \rightarrow J \text { in } \Sigma_{A^{*}}
\end{aligned}
$$

This is irreducible because $\Sigma_{A}$ and $\Sigma_{F}$ are. Define a $\mathbb{Z} \otimes_{T} G$ action on this by:

$$
h(I) \text { is defined as on } L_{A}, h(I, g, n)=\left(h(I),\left(T^{p-n+1} h\right) g, n\right) .
$$

Notice that for a fixed $g$ the symbols $\{(I, g, n)\}, I \in L_{F}, 0 \leq n<p$ and all transitions among them define an irreducible ssft $\Sigma_{F}^{g} \subseteq \Sigma_{\bar{A}}$. It is a standard $p$ periodic cover of $\Sigma_{F}$. Let $\Sigma_{F}^{(g, n)}$ be the cyclic subset of $\Sigma_{F}^{g}$ consisting of all points with $(I, g, n)$ for $I \in L_{F}$ in the time zero coordinate, then $\sigma\left(\Sigma_{F}^{(g, n)}\right)=\Sigma_{F}^{(g, n+1)} \bmod p$ and $\Sigma_{F}^{g}=\Sigma_{F}^{(g, 0)} U$ $\cdots \cup \Sigma_{F}^{(g, p-1)}$. Let $L_{F}^{(g, n)}=\left\{(I, g, n): I \in L_{F}\right\}$, and observe that only $L_{F}^{(g, 0)}$ has successors outside of $L_{F}^{g}$. We have a well-defined $\mathbb{Z} \otimes_{T} G$ action on $\Sigma_{\bar{A}}$ because of this and because if $(I, g, n) \rightarrow(J, g, n+1)$ in $\Sigma_{F}^{g}$ then $h(I, g, n) \rightarrow T h(J, g, n+1)$ in $\Sigma_{F}^{k}$ where $k=\left(T^{p-n+1} h\right) g$. The obvious map $\Sigma_{\bar{A}} \rightarrow \Sigma_{A}$ is left resolving, one-to-one a.e., $p|G|$-to-one at most and commutes with the $\mathbb{Z} \otimes_{T} G$ actions. Again, every element of $G(K)$ is a magic word, but now the inverse image of $\Sigma_{F}$ is $|G|$ copies of the standard $p$ periodic cover of $\Sigma_{F}$.

From this point on we will assume that all actions are special.

Example 4. Define $\Sigma_{\bar{A}}$ by the first of the two graphs that follow, and $\Sigma_{A}$ by the second. Then the symbol $K$ in the previous proof can be taken to be $1_{(1,2)}$ in $L_{A}$, and $\Sigma_{F} \subseteq \Sigma_{A}$ will be the single fixed point of all 2's. Using these, the construction in the proof will produce $\Sigma_{\bar{A}}$.
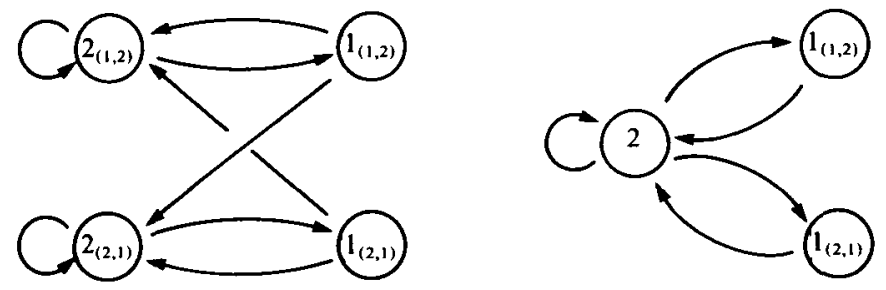

3. Classification of $\mathbb{Z} \otimes_{T} G$ action; the aperiodic case In this section we will prove the following:

THEOREM 4. If $\Sigma_{A}$ and $\Sigma_{B}$ are aperiodic ssft, $G$ is a finite group, $T$ is an automorphism of $G$, and $\Sigma_{A}$ and $\Sigma_{B}$ both have $\mathbb{Z} \otimes_{T} G$ actions, then these actions are almost topologically conjugate if and only if the two ssft have the same topological entropy. 
The proof is a slight generalization of the proof of the corresponding thorem in [1]. There are five parts: (i) getting a suitable positive integer matrix $F$ satisfying $A F=F B$, (ii) defining the tableau, (iii) filling in the tableau to get magic words when $\Sigma_{A}$ and $\Sigma_{B}$ have fixed points, (iv) showing that every aperiodic ssft is almost topologically conjugate to an ssft with a fixed point, $(\mathrm{v})$ building a 'tower' to see that (iii) and (iv) together imply the theorem. In each section we will first explain what is needed for the $\mathbb{Z}$ action alone and then show what changes are needed to deal with the $\mathbb{Z} \otimes_{T} G$ action. The difference is that any symbolic manipulation must be pushed around by the group action.

Part (i).

Lemma (Furstenberg). Let $A$ and $B$ be irreducible transition matrices. $\lambda_{A}=\lambda_{B}$ if and only if there exists a positive integral matrix $F$ such that $A F=F B$.

Proof. (Note: this is not the proof in [1], it is the original one given by Furstenberg and can be found in [5] - the reason we use this proof here is that it allows us to construct an $F$ with some additional properties.) Suppose $\lambda_{A}=\lambda_{B}$. Choose a strictly positive right eigenvector, $\imath$, for $A$, and a strictly positive left eigenvector, $\ell$, for $B$. Let $N=\imath \ell$ (matrix multiplication). It is a positive real matrix satisfying $A N=N B$. For $\varepsilon>0$ choose a $t \in \mathbb{R}^{+}$such that $t N=F+U$ where $F$ is a strictly positive integer matrix and $U$ has all entries less than $\varepsilon$ in absolute value. Then $A F-F B$ is an integer matrix. But

$$
|A F-F B| \leq|A(F-t N)|+|(t N-F) B| \leq(|A|+|B|)|U| .
$$

By choosing $\varepsilon$ sufficiently small we get that $A F-F B=0$.

In [1] any choice of $F$ will do; in this case we must be more careful. The extra requirement is spelled out in the following lemma.

LEMMA. Let $\Sigma_{A}, \Sigma_{B}$ be irreducible ssft. Suppose $\lambda_{A}=\lambda_{B}$ and they have $\mathbb{Z} \otimes_{T} G$ actions. Then there is a positive integral $F$ such that $A F=F B$ and $F_{I, J}=F_{g l, h J}$ for all $g, h \in G$.

Proof. Recall the construction of $F$ in the previous lemma. $F \approx t \imath \ell$ where $t \in \mathbb{R}^{+}$, $A \ell=\lambda \ell$, and $\ell B=\lambda \ell$. The extra condition is satisfied because a $\mathbb{Z} \otimes_{T} G$ action means $P_{g} A=A P_{T_{g}}$ and $Q_{g} B=B Q_{T g}$ for the appropriate families of permutation matrices $\left\{P_{h}\right\},\left\{Q_{h}\right\}$ as defined in observation 2. This means $P_{T^{-n}{ }_{g}} A^{n}=A^{n} P_{g}$; since $T^{p}=\mathrm{id}$ for some $p>0, P_{g} A^{p}=A^{p} P_{g}$ and $\lambda^{p} P_{g} \imath=P_{g} A^{p} \imath=A^{p} P_{g}$, so $P_{g} \imath=\imath$, for all $g \in G$. The same is true for $B, \ell Q_{g}=\ell$ and since $F \approx t_{z} \ell$ we have the desired result.

Part (ii). Here we define the tableau and then show that it can be filled in so that we have a common finite-to-one extension for the special $\mathbb{Z} \otimes_{T} G$ actions. The tableau is defined just as it was in [1]. Take $A$ and $B$ to be irreducible transition matrices with a common maximal eigenvalue. Suppose they have alphabets $L_{A}=\left\{1, \ldots,\left|L_{A}\right|\right\}$ and $L_{B}=\left\{1, \ldots,\left|L_{B}\right|\right\}$ and that we have chosen a positive integral matrix $F$ satisfying $A F=F B$. Define a new symbol set

$$
L_{C}=\left\{(I, J, K): I \in L_{A}, J \in L_{B}, 1 \leq K \leq F_{I J}\right\} .
$$

We call the following list of partially assigned transitions the tableau associated with $A, B$ and $F$. 
For each $I \in L_{A}$ we get a page in the tableau, and for each $J \in L_{B}$ we get a paragraph on each page. Pick $I \in L_{A}$, its page is:

\begin{tabular}{|c|c|c|c|c|}
\hline$(I, 1,1)$ & $\rightarrow$ & $\left(\cdot, 1_{1}, \cdot\right)$ & $\cdots$ & $\left(\cdot, 1_{|f(1)|}, \cdot\right)$ \\
\hline$\left(I, 1, F_{I 1}\right)$ & $\rightarrow$ & $\left(\cdot, 1_{1}, \cdot\right)$ & $\cdots$ & $\left(\cdot, 1_{|f(1)|}, \cdot\right)$ \\
\hline$(I, J, 1)$ & $\rightarrow$ & $\left(\cdot, J_{1}, \cdot\right)$ & $\cdots$ & $\left(\cdot, J_{|f(J)|}, \cdot\right)$ \\
\hline$\left(I, J, F_{H}\right)$ & $\rightarrow$ & $\left(\cdot, J_{1}, \cdot\right)$ & $\cdots$ & $\left(\cdot, J_{|f(J)|}, \cdot\right)$ \\
\hline$\left(I,\left|L_{B}\right|, 1\right)$ & $\rightarrow$ & $\left(\cdot,\left|L_{B}\right|_{1}, \cdot\right)$ & $\cdots$ & $\left(\cdot,\left|L_{B}\right|_{\left|f\left(\left|L_{B}\right|\right)\right|}, \cdot\right)$ \\
\hline$\left.,\left|L_{B}\right|, F_{I\left|L_{B}\right|}\right)$ & $\rightarrow$ & $\left(\cdot,\left|L_{B}\right|_{1}, \cdot\right)$ & $\cdots$ & $\left(\cdot,\left|L_{B}\right|_{\left|f\left(\left|L_{B}\right|\right)\right|}\right.$ \\
\hline
\end{tabular}

The paragraph corresponding to a $J \in L_{B}$ is composed of the rows where $J$ appears as the middle symbol of the left most entry.

If we see in the tableau $(I, J, K) \rightarrow\left(\cdot, J^{\prime}, \cdot\right)$ we have by definition that $J \rightarrow J^{\prime}$ is a $B$-transition. We want to fill in the first component on the right with an $I^{\prime}$ for which $I \rightarrow I^{\prime}$ is an $A$-transition and the third component with a $K^{\prime}, 1 \leq K^{\prime} \leq F_{I^{\prime} J^{\prime}}$ such that the resulting $\left(I^{\prime}, J^{\prime}, K^{\prime}\right)$ is used only once in the page for $I$. We also require that each $\left(I^{\prime}, J^{\prime}, K^{\prime}\right)$ that can appear in the page for $I$ does appear. That this can be done is a consequence of the matrix equation $A F=F B$.

Generally, there are many choices for filling in the tableau. Once the page is filled in for each $I \in L_{A}$ we have defined a tansition matrix $C$ for the symbols $L_{C}$. We define one-block mappings, $\pi$ and $\bar{\pi}$ from $\Sigma_{C}$ to $\Sigma_{A}$ and $\Sigma_{B}$ by $\pi(I, J, K)=I$ and $\bar{\pi}(I, J, K)=J$. Because of the construction of the tableau $\pi$ is left resolving and $\bar{\pi}$ is right resolving. $\Sigma_{C}$ may not be irreducible, but if there are resolving blocks for both $\pi$ and $\bar{\pi}$ it will be. This follows from the fact that one of the maps is left resolving and the other right.

Now suppose $\Sigma_{A}$ and $\Sigma_{B}$ have $\mathbb{Z} \otimes_{T} G$ actions. We want to show that a tableau can be produced and filled in so that the resulting $\Sigma_{C}$ has a $\mathbb{Z} \otimes_{T} G$ action that is a continuous extension of the $\mathbb{Z} \otimes_{T} G$ actions on $\Sigma_{A}$ and $\Sigma_{B}$. First choose $F$ as described in the second lemma of part one. Here we will use the condition that $F_{I J}=F_{g I h J}$ all $g, h \in G$. Write down the tableau as before. Notice that if we examine the page for $I$, it looks just like the ones for $g I$, each $g \in G$. That is, for each row in $I$ 's page

$$
(I, J, K) \rightarrow\left(\cdot, J_{1}, \cdot\right)\left(\cdot, J_{2}, \cdot\right) \cdots\left(\cdot, J_{|f(J)|}, \cdot\right)
$$

there is a corresponding one in $g(I)$ 's page

$$
(g(I), g(J), K) \rightarrow\left(\cdot, \operatorname{Tg}\left(J_{1}\right), \cdot\right)\left(\cdot, \operatorname{Tg}\left(J_{2}\right), \cdot\right) \cdots\left(\cdot, \operatorname{Tg}\left(J_{|f(J)|}\right), \cdot\right) .
$$

The method for filling in the tableau to produce the desired $G$ action on $\Sigma_{C}$ is obvious. $L_{A}$ is partitioned into disjoint $G$ orbits. Choose one representative from each. Fill in the page for each of these representatives. Then use $G$ to push these 
to the rest of the tableau. So if we have $(I, J, K) \rightarrow\left(I^{\prime}, J^{\prime}, K^{\prime}\right)$ we fill in $(g(I), g(J), K) \rightarrow\left(T g(I), T g(J), K^{\prime}\right)$. Since $F_{I J}=F_{g I h J}$ everything fits. This produces a $\Sigma_{C}$ with the desired $\mathbb{Z} \otimes_{T} G$ action. The maps $\pi$ and $\bar{\pi}$ take this action to the ones on $\Sigma_{A}$ and $\Sigma_{B}$.

Part (iii). This is the crucial step. Here we assume that $\Sigma_{A}$ and $\Sigma_{B}$ have fixed points and show that the tableau can be filled in so that the resulting maps are one-to-one a.e. This is done by carefully filling in the tableau so that resolving blocks are produced for both maps. This ensures that $\Sigma_{C}$ is irreducible and that the maps are one-to-one a.e. As before, we will present the argument worrying only about the shift, then show how it can be modified to take into account the entire $\mathbb{Z} \otimes{ }_{T} G$ actions.

Suppose $A$ and $B$ are irreducible transition matrices with fixed states. The symbol sets are of the form $L_{A}=\left\{1, \ldots,\left|L_{A}\right|\right\}, L_{B}=\left\{1, \ldots,\left|L_{B}\right|\right\}$. In both cases assume 1 is the fixed state. In the directed graph defined by $A$ we 'grow' a tree rooted on 1 of simple $\boldsymbol{A}$-paths which can only join but not cross each other, leading from any $I \in L_{A}, I \neq 1$, forward to 1 . For each $I$ denote the tree transition by $I \rightarrow I_{1}$ and the non-tree ones by $I \rightarrow I_{2}, \ldots, I_{|f(I)|}$. Let $I_{1}=1$ when $I=1$. Likewise in the directed graph determined by $B$ grow a tree rooted on 1 of simple non-crossing $B$-paths from each $J \in L_{B}$ backwards to 1 . These tree transitions are denoted by $J_{1}^{*} \rightarrow J$, with $J_{1}^{*}=J$ for $J=1$, and the non-tree ones by $J_{2}^{*}, \ldots, J_{|p(J)|}^{*} \rightarrow J$. Next we will sequentially fill in the tableau in five steps. When making an assignment in this procedure, say, $(I, J, K) \rightarrow\left(I^{\prime}, J^{\prime}, K^{\prime}\right)$ two conditions must prevail in order to fill in the tableau legally:

(i) no $\left(\cdot, J^{\prime}, \cdot\right)$ has previously been assigned as a successor to $(I, J, K)$;

(ii) $\left(I^{\prime}, J^{\prime}, K^{\prime}\right)$ has not been previously assigned as a successor to any $(I, \cdot, \cdot)$. As the following steps are presented we leave it to the reader to check that these conditions are met. $I$ and $J$ will be used as generic symbols of $L_{A}$ and $L_{B}$, respectively.

Step 1. $I \neq 1, J=1$, set

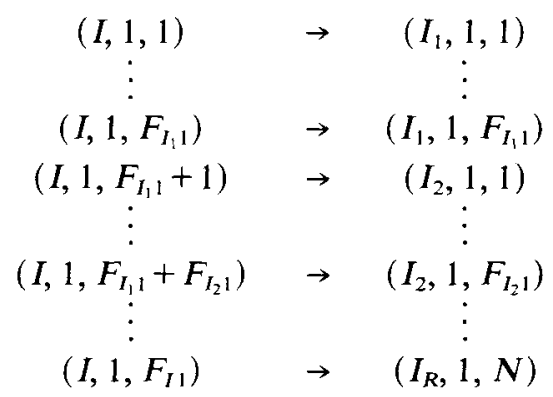

where $1 \leq R \leq|f(I)|$ and $N$ is whatever positive integer is needed.

Step 2. For $I=1, J=1$, (assume $F_{11}>1$ ) set

$$
\begin{array}{ccc}
(1,1,1) & \rightarrow & (1,1,1) \\
(1,1,2) & \rightarrow & (1,1,1) \\
(1,1,3) & \rightarrow & (1,1,2) \\
\vdots & & \vdots \\
\left(1,1, F_{11}\right) & \rightarrow & \left(1,1, F_{11}-1\right)
\end{array}
$$


The idea of step 1 is to proceed from $(I, 1, K)$ by forward transitions in $C$ in which, if possible, the first coordinate moves along tree transitions while the third stays constant as 1 is being repeated successively in the second. If this is not possible, the first coordinate takes a non-tree transition and the third is reduced. Eventually either the third coordinate is reduced to 1 or we arrive at $(1,1, K)$. In step 2 , if $1<K \leq F_{11}$, we spiral down from $(1,1, K)$ to $(1,1,2)$ and then out to $\left(1_{2}, 1,1\right)$. Then every $(I, 1,1)$ leads to $(1,1,1)$ along tree transitions as 1 is repeated in the second coordinate. Then no matter how the rest of the tableau is filled in the block $B=[1,1, \ldots, 1]$ with 1 repeated $\left|L_{C}\right|$ times, maybe even less, is resolving for $\bar{\pi}$ - i.e. all $C$-admissible $\left|L_{C}\right|$-blocks in $\bar{\pi}^{-1} B$ are of the form $[(I, 1, K), \ldots,(1,1,1)]$.

Step 3. For $I=1, J \neq 1$, set

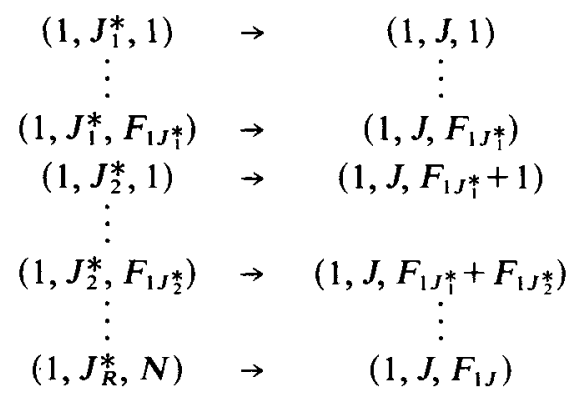

where $1 \leq R \leq|p(J)|$ and $N$ is whatever positive integer is needed.

Step 4. Assuming $F_{11}>1$ set

$$
\left(1,1_{2}^{*}, 1\right) \rightarrow\left(1,1, F_{11}\right) \text {. }
$$

Step 5. Fill in the rest of the tableau arbitrarily but according to the rules of part 2.

Now moving from $(1, J, K)$ by backward transitions in $C$ the second coordinate according to step 3 moves back along tree transitions when possible while keeping the third coordinate fixed as 1 is repeated in the first. On the other hand if this is not possible the second coordinate takes a non-tree transition and the third is reduced. Eventually either the third coordinate is reduced to 1 or we arrive at $(1,1, K)$. If $1<K \leq F_{11}$, by steps 2 and 4 we spiral back up from $(1,1, K)$ to $\left(1,1, F_{11}\right)$ and then back to $\left(1,1_{2}^{*}, 1\right)$. Then $(1, J, 1)$ leads back to $(1,1,1)$ along tree transitions as 1 is repeated in the first coordinate. Then no matter how the rest of the tableau is filled in by step 5 , the block $B=[1, \ldots, 1]$ of $\left|L_{C}\right|$ consecutive 1's is resolving for $\pi$-i.e. all $C$-admissible $\left|L_{C}\right|$-blocks in $\pi^{-1}(B)$ are of the form $[(1,1,1), \ldots,(1, J, K)]$. In the case $F_{11}=1$ replace $F$ by $2 F$.

At this point we have shown that if $A$ and $B$ are irreducible transition matrices with positive trace and the same largest eigenvalues then $\Sigma_{A}$ and $\Sigma_{B}$ are almost conjugate factors of $\Sigma_{C}$. 
Let $I=g(1)$; it need not be a fixed symbol. If $T g \neq g$ then $g(1) \neq T g(1)$ since we are dealing with a special action. For each $I \in G(1)$ denote by $I \rightarrow I_{\alpha}$ (or $I_{\alpha}^{*} \rightarrow I$ ) the special transition where $I=g(1)$ and $I_{\alpha}=T g(1)$ (or $I_{\alpha}^{*}=T^{-1} g(1)$ ). For a $g \in G$ let $s$ be the least integer so that $T^{s} g=g$. Taking $I_{\alpha^{s}}=T^{s} g(1)$ we have the loop $I \rightarrow I_{\alpha} \rightarrow I_{\alpha^{2}} \rightarrow \cdots \rightarrow I_{\alpha^{s-1}} \rightarrow I$. To complete this part of the argument we must choose our trees carefully, taking into account these symbols $I \in G(1)$. The extra condition on our trees is spelled out in the following lemma.

Leмma. Suppose $A$ is an aperiodic transition matrix with non-zero trace, $G$ is a finite group, and $\mathbb{Z} \otimes_{T} G$ acts on $\Sigma_{A}$. Then there is a $\Sigma_{\bar{A}}$, with a conjugate $\mathbb{Z} \otimes_{T} G$ action, where we can choose a tree in the graph of $\bar{A}$, based on a fixed symbol, 1 , so that for $J \in G(1), J \neq 1 ; J \neq I_{1}$ for any $I \in L_{\bar{A}}$.

Proof. Begin with the graph of $A$ where 1 is the fixed symbol, by using the elementary conjugacies we can easily make sure that 1 has exactly one predecessor and one successor other than itself, and that neither is in the $G$-orbit of 1 .

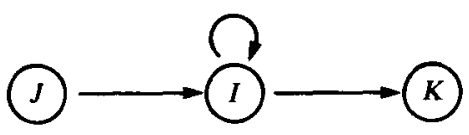

This means that if we look at any $\alpha$ loop in $G(1)$, it looks like:

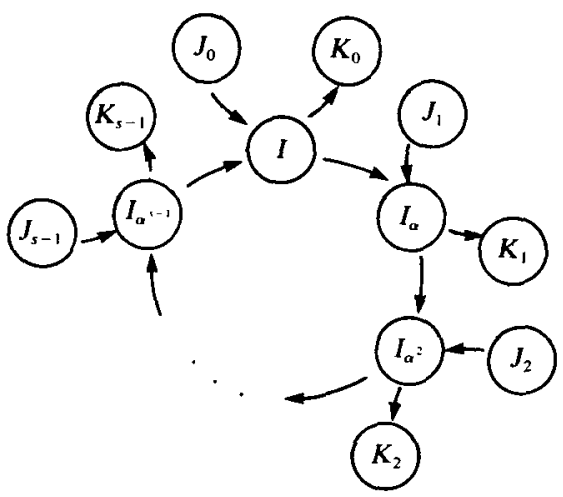

where $J_{t}$ and $K_{t}$ are not in $G(1)$ for all $t$.

First we will show that we can make sure there are no tree transitions into the $\alpha$ loop from the outside. Assume $J_{0} \rightarrow I$ is a tree transition, and that $I \rightarrow I_{\alpha}$, $I_{\alpha} \rightarrow I_{\alpha^{2}}, \ldots, I_{\alpha^{n-1}} \rightarrow I_{\alpha}{ }^{n}, I_{\alpha^{n}} \rightarrow K_{n}$ are also tree transitions for some $n, 1 \leq n \leq s-1$. We will show by induction that we can get rid of this. Split each element in $G(1)$ by predecessors, so in particular $I_{\alpha^{\prime}}$ gets split into $\left[I_{\alpha^{1-1}}, I_{\alpha^{\prime}}\right]$ which we will again call $I_{\alpha}$ and $\left[J_{t}, I_{\alpha^{1}}\right]$ which we will now call $J_{t+1}^{1}$. This results in a new conjugate aperiodic ssft, that inherits a tree and a $\mathbb{Z} \otimes_{T} G$ action. The $\alpha$ loop now looks like: 


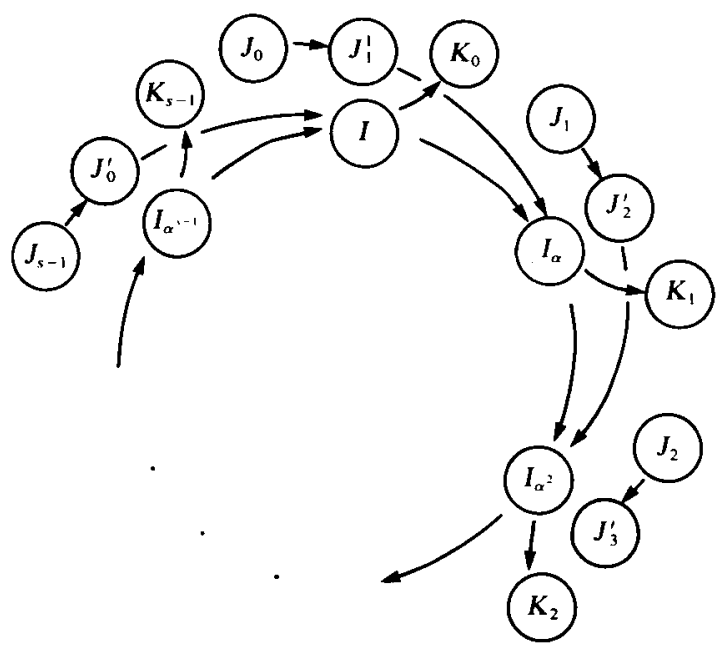

Notice what we have accomplished. Before splitting we had a tree transition enter the loop proceed along the loop for $n$ steps and then exit. Now the transition enters, proceeds for $n-1$ steps and exits. By repeating this construction a sufficient number of times we may assume no tree transition enters an $\alpha$ loop from the outside.

It may still be that there are tree transitions that agree with $\alpha$ transitions. Notice that because of the previous construction we can throw out all of $G(1)$, except 1 , and the remaining graph will still contain a tree leading from each vertex, forward to 1 . This means that in the whole graph if $I \rightarrow I_{\alpha}$ is a tree transition, we can change $I$ 's tree transition to $I \rightarrow J$ where $J$ is $I$ 's successor outside of $G(1)$, and we will still have a tree. Do this for all $I \in G(1), I \neq 1$ and we have the desired result.

We are now in a position to complete this part of the proof. Repeat the procedure of step 1 , but this time only for those $I$ 's not in the orbit of 1 . Call these original transitions. Next fill in the transitions forced by $G$. If $(I, J, K) \rightarrow\left(I^{\prime}, J^{\prime}, K^{\prime}\right)$ is an original transition then $(g I, g J, K) \rightarrow\left(T g\left(I^{\prime}\right), T g\left(J^{\prime}\right), K^{\prime}\right)$ is a forced transition for each $g \in G$. We must see that no contradictions have arisen. First, notice that the pages for $I \in G(1) \subseteq L_{A}$, are still blank. Next examine the page of an $I \notin G(1)$. Notice that only those paragraphs corresponding to a $J \in G(1) \subseteq L_{B}$ have any rows with any transitions filled in. In fact, each row in such a paragraph has exactly one transition filled in because there is a unique $h \in G$ such that $h J=1$. At this point there are no contradictions.

Step 2. This is now for $I \in G(1) \subseteq L_{A} ; J=1$, (note that $F_{I 1}=F_{11}$ ) set

$$
\begin{array}{ccc}
(I, 1,1) & \rightarrow & \left(I_{1}, 1,1\right) \\
(I, 1,2) & \rightarrow & \left\{\begin{array}{cc}
\left(I_{\alpha}, 1,1\right) & \text { if } I \neq 1 \\
\left(I_{2}, 1,1\right) & \text { if } I=1
\end{array}\right. \\
(I, 1,3) & \rightarrow & \left(I_{\alpha}, 1,2\right) \\
\vdots & & \vdots \\
\left(I, 1, F_{11}\right) & \rightarrow & \left(I_{\alpha}, 1, F_{11}-1\right) .
\end{array}
$$


These are original transitions and the ones forced by $G$ are filled in as in step 1 . We must again check for contradictions. First notice that the pages affected in step 1 and those affected here are disjoint. No contradictions can arise from conflict between the two steps. Step 2 is internally consistent by the same reasoning as applied in step 1.

At this point we have constructed a resolving block for $\bar{\pi}$. The reasoning is identical to that after step 2 in the original argument except that $(I, 1, K)$ for $I \in G(1), K \geq 2$ proceeds by forward transitions down to $\left(I_{\alpha}{ }^{K-2}, 1,2\right)$ as 1 is repeated in the second coordinate. Then if $I \neq 1$, and another 1 is repeated in the second coordinate, $\left(I_{\alpha^{\kappa-1}}, 1,1\right)$ results. If $I=1$, another transition results in $\left(1_{2}, 1,1\right)$. In either case we are led to $(1,1,1)$ as 1 is repeated in the second coordinate.

Step 3. For $I=1, J \notin G(1) \subseteq L_{B}$ the transitions are filled in just as in the original argument. Recall that the only lines where any transitions are filled in are in the paragraphs with $J \in G(1)$ on pages with $I \notin G(1)$ from step 1 and in the paragraphs with $J \in G(1)$ on pages with $I \in G(1)$ from step 2 . In step 3 we have filled in transitions in rows in paragraphs for arbitrary $J$, on pages with $I \in G(1)$. We must see that no contradictions arise from step 2 . Recall that in the transitions from step $2,(I, J, K) \rightarrow\left(I^{\prime}, J^{\prime}, K^{\prime}\right)$, all have $J^{\prime} \in G(1)$. The transitions from step 3 never have $J^{\prime} \notin G(1)$. There are no contradictions in step 3 arising from either step 1 or step 2. Step 3 is internally consistent by the same reasoning as applied in steps 1 and 2 .

Step 4. For $J \in G(1),\left(F_{1 J}=F_{11}\right)$, set

$$
\begin{array}{ll}
\left(1, J_{1}^{*}, 1\right) \rightarrow\left(1, J, F_{11}\right) & \text { if } J \neq 1, \\
\left(1, J_{2}^{*}, 1\right) & \text { if } J=1 .
\end{array}
$$

Since the transitions arising here all have $I \in G(1)$ the only possible external contradictions are with either step 2 or step 3 . When $J \neq 1, J_{1}^{*} \notin G(1)$, so here the only possible contradiction is with step 3 . It may be that there is a $J^{\prime} \notin G(1)$ with $\left(J^{\prime}\right)_{n}^{*}=J_{1}^{*}$ so that in step 3 we have $\left(1,\left(J^{\prime}\right)_{n}^{*}, 1\right)=\left(1, J_{1}^{*}, 1\right) \rightarrow\left(1, J^{\prime}, K\right)$ and in step 4 we have $\left(1, J_{1}^{*}, 1\right) \rightarrow\left(1, J, F_{11}\right)$ but since $J^{\prime} \notin G(1)$ and $J \in G(1)$ there won't be a contradiction. When $J=1$ we have

$$
\left(1,1_{2}^{*}, 1\right) \rightarrow\left(1,1, F_{11}\right) \text {. }
$$

Since $1_{2}^{*} \notin G(1)$ the same reasoning applies here. Finally, we check to see that there are no internal contradictions in step 4 . This is the same as steps 1-3.

Now we have constructed a resolving block for $\pi$. It works just as it did in the original argument with one exception. If we are at $(1, J, K)$ for $J \in G(1)$ and move backward as 1 is repeated in the first coordinate, $J$ takes successive $J_{\alpha}^{*}$ transitions, $K$ is increased until it reaches $F_{11}$. Then we go to a $\left(1, J^{\prime}, 1\right)$ and then by tree transitions to $\{1,1,1)$, never seeing another $J \in G(1)$ as a centre coordinate.

Step 5. Fill in the rest of the tableau arbitrarily but according to the rules of part 2 to get a $\mathbb{Z} \otimes_{T} G$ action on $\Sigma_{C}$ that commutes with the maps $\pi$ and $\bar{\pi}$.

Part (iv). Here we will show that any aperiodic ssft with a $\mathbb{Z} \otimes_{T} G$ action is almost-topologically conjugate to $\mathrm{a} \mathbb{Z} \otimes_{T} G$ action on an ssft with a fixed point under $\sigma$. This does not follow the proof of the corresponding statement in [1]. Instead it 
follows proofs of that statement done independently by W. Krieger [4] and $M$. Keane. The idea is fairly straightforward, we begin with $A$ and find a conjugate representation whose graph contains a simple cycle all of whose vertices have a common predecessor and disjoint successors. This is done by splitting states and making amalgamations. This ssft can then be covered, with an almost-conjugate extension, by making two copies of this cycle, each with the same successors but dividing the predecessors. Each vertex in one copy will have only the single common predecessor outside of the cycle. The corresponding vertex in the other copy will have all the other predecessors. The map obtained by identifying the two cycles is one-to-one a.e. and right resolving. Finally, this extension has an almost conjugate factor obtained by collapsing the copy of the cycle, where each vertex has the common predecessor, to a single fixed vertex. This is a one-to-one a.e. left resolving map whose image is the desired ssft with a fixed point. Our proof here follows this outline. The problem is to make sure that all these operations can be made consistent with the $\mathbb{Z} \otimes_{T} G$ action. We begin with the following lemma.

LEмMA. Given $a \mathbb{Z} \otimes_{T} G$ action there is a conjugate $\mathbb{Z} \otimes_{T} G$ action for which the graph of the ssft contains a simple p-cycle

$$
J^{0} \rightarrow J^{1} \rightarrow J^{2} \rightarrow \cdots \rightarrow J^{p-1} \rightarrow J^{0},
$$

and there exists an integer $N$, a state $J_{0}$ and allowable paths $\gamma_{R}=\left[J_{0}^{R}, J_{1}^{R}, \ldots, J_{N}^{R}\right]$, $0 \leq R<p$ such that

(1) each $J_{0}^{R}=J_{0}, J_{N}^{R}=J^{R}$, and

(2) $\left\{J_{i}^{R}\right\}, 0 \leq R<p, 0 \leq i \leq N$ are all in distinct $G$-orbits.

Note. After proving this, we will then show that in fact $N$ can be chosen to be one.

Proof. First, we show that there is a $\sigma$-periodic point, $x$, such that $g(x) \notin \sigma$-orb $(x)$ for all $g \neq$ id. In fact, any periodic point with least period $p$, for $p$ prime and greater than the order of $G$ has this property. Let $k$ be the period of $T$; then $\sigma^{k} \circ g=g \circ \sigma^{k}$ for all $g \in G$. For a point, $x$, with prime period $p, p>k, \sigma$-orb $(x)=\sigma^{k}$-orb $(x)$. Suppose for some $g \neq$ identity, $g(x) \in \sigma$-orb $(x)$. Then $g(x) \in \sigma^{k}$-orb $(x)$ so $g(x)=$ $\sigma^{m k}(x)$ for some $0 \leq m<p$. Let $j$ be the order of $g$. We have $x=g^{j}(x)=\sigma^{j m k}(x)$, so $p$ divides $j m k$, since $j \neq 0$ and is less than $p, p$ divides $m k$, but this means $g(x)=x$. This contradicts the fact that the $\mathbb{Z} \otimes_{r} G$ action is special. For $x$ of prime period greater than the order of $G$, and $g \neq$ identity, $g(x) \notin \sigma$-orb $(x)$. Choose such an $x$, then by going to a sufficiently high block system we can assume that the $\sigma$-orbit of $x$ is represented by a simple $p$-cycle,

$$
I^{0} \rightarrow I^{1} \rightarrow \cdots \rightarrow I^{p-1} \rightarrow I^{0},
$$

in the graph of $A$. Also that for $g \neq$ identity $g\left(\left\{I^{R}\right\}_{0 \leq R<p}\right) \cap\left\{I^{R}\right\}_{0 \leq R<p}=\varnothing$. Since $A$ is aperiodic, we can find an $n>0$, an $I_{0} \in f\left(I^{0}\right)$, and paths $\gamma_{R}=\left[I_{0}^{R}, I_{1}^{R}, \ldots, I_{n}^{R}\right]$, $0 \leq R<p$, where:

$I_{0}^{R}=I_{0}, I_{n}^{R}=I^{R}$ for all $R$;

$\left\{I_{j}^{R}\right\}_{0 \leq R<p, 1 \leq j \leq n}$ are distinct;

$\left\{I_{j}^{R}\right\}_{0 \leq R<p, 0 \leq j<n}$ all miss the $G$-orbit of $\left\{I^{R}\right\}_{0 \leq R<p}$. 
It may be necessary to go to an even higher block presentation to do this. Let $\left[K_{0}, \ldots, K_{n-1}\right]=\left[I^{p-n+1}, \ldots, I^{p-1}, I^{0}\right]$ (where $I^{p-t} \equiv I^{p-t(\bmod p)}$ if $p-t$ is negative). For $0 \leq R<p$, and $0 \leq t \leq 2 n$ let

$$
J_{t}^{R}= \begin{cases}{\left[K_{t}, \ldots, K_{n-1}, I_{0}^{R}, \ldots, I_{t}^{R}\right]} & 0 \leq t<n \\ {\left[I_{t-n}^{R}, \ldots, I_{n}^{R}, I^{R+1}, \ldots, I^{R+t-n}\right]} & n \leq t \leq 2 n\end{cases}
$$

(where $I^{s} \equiv I^{s(\bmod p)}$ for $\left.s \geq p\right)$. This defines symbols, $J_{l}^{R}$, in the $(n+1)$-block presentation. The periodic point $x$ is represented by the cycle

$$
J^{0} \rightarrow J^{1} \rightarrow \cdots \rightarrow J^{p-1} \rightarrow J^{0}
$$

where $J^{R}=\left[I^{R}, I^{R+1}, \ldots, I^{R+n}\right]$. Now let $N=2 n$ and $\gamma_{R}=\left[J_{0}^{R}, \ldots, J_{N}^{R}\right]$ and we will see that the $\gamma_{R}$ satisfy conditions (1) and (2) of the lemma. Condition (1) is clearly satisfied. To see that (2) is satisfied, observe that since each $J_{1}^{R}, 0 \leq t<n$, begins with an $I^{k}$; these have disjoint $G$-orbits by the conditions imposed on $\left\{I^{0}, \ldots, I^{p-1}\right\}$. By the same reasoning each $J_{t}^{R}, n \leq t \leq 2 n$, ends with an $I^{k}$; so these have disjoint $G$-orbits. Since each $J_{t}^{R}, 0 \leq t<n$ ends with an $I_{s}^{R}, s<n$, and each $J_{t}^{R}, n \leq t \leq 2 n$ ends with an $I^{k}$ the condition that $\left\{I_{j}^{R}\right\}_{0 \leq R<p, 0 \leq j \leq n}$ all miss the $G$-orbit of $\left\{I^{0}, \ldots, I^{p-1}\right\}$ means that the $G$-orbits of the $J_{t}^{R}$ with $t \geq n$ and the $J_{t}^{R}$ with $t<n$ are disjoint. This means condition (2) is satisfied.

Next, by induction, we show that $N$ can be chosen to be one. The rough idea is that we would like to amalgamate the $\left\{J_{1}^{R}\right\}, 0 \leq R<p-1$. But as it stands these states don't satisfy the hypotheses for amalgamation. We need some preliminary splitting. Let

$$
E_{1}=\left\{J_{2}^{0}\right\}, \quad E_{2}=f\left(J_{1}^{0}\right)-\left\{J_{2}^{0}\right\} .
$$

This is a partition of $f\left(J_{1}^{0}\right)$ and this describes a state splitting as described earlier. Recall in the discussion of state splitting the way this is pushed around by the $G$ action. Condition (2) of the previous lemma makes sure that this doesn't affect any of the other $J_{i}^{R}$. This replaces $J_{1}^{0}$ by a new (split state) $J_{1}^{0}$ and keeps all other conclusions of the lemma in effect. Do this for each $R$. The result is that each new $J_{1}^{R}$ has exactly one successor, namely $J_{2}^{R}$, and the conclusions of the lemma still hold. Now split by predecessors so that each new $J_{1}^{R}$ has exactly one predecessor $J_{0}^{R}$ and one successor $J_{2}^{R}$. We can now amalgamate the $\left\{J_{1}^{R}\right\}$. The new conjugate ssft still has a special $\mathbb{Z} \otimes_{T} G$ action, and the lemma is still satisfied but the length of the paths has been reduced to $N-1$. This means that up to topological conjugacy we may assume that we have special $\mathbb{Z} \otimes_{T} G$ action and that somewhere in the graph we see the picture:

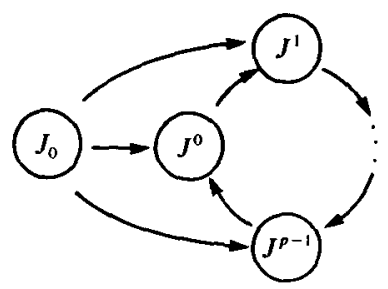

and the $\left\{J_{0}, J^{0}, \ldots, J^{p-1}\right\}$ are in disjoint $G$-orbits. 
By splitting states we can easily guarantee that the $\left\{J^{R}\right\}, 0 \leq R<p$ have disjoint follower sets.

Next we build an almost conjugate extension, $\Sigma_{B}$, in which somewhere in the graph of $\Sigma_{B}$ we have the above picture and also

$$
p\left(J^{R}\right)=\left\{J_{0}, J^{R-1}\right\} .
$$

To produce $\Sigma_{B}$ let

$$
L_{B}=L_{A} \cup\left\{K_{g}^{R}\right\}, \quad 0 \leq R<p, g \in G .
$$

Eliminate the transitions

$$
T^{-1} g\left(J_{0}\right) \rightarrow g\left(J^{R}\right), \quad g \in G, 0 \leq R<p,
$$

from those allowed by $A$ and add new transitions

(i) each $T^{-1} \mathrm{~g}\left(J_{0}\right) \rightarrow K_{\mathrm{g}}^{R}$;

(ii) for $L \in f\left(J^{R}\right), L \neq J^{R+1}$, let $K_{g}^{R} \rightarrow T g(L)$;

(iii) $K_{g}^{R} \rightarrow K_{T_{g}}^{R+1}$.

Now observe that the cycle

$$
K_{e}^{0} \rightarrow K_{e}^{1} \rightarrow \cdots \rightarrow K_{e}^{p-1} \rightarrow K_{e}^{0} \quad(e \text { is the group identity element), }
$$

satisfies conditions (1), (2), (3), and (4) with $J_{0}$ unchanged but each $J^{R}$ is replaced by $K_{e}^{R}$. The $G$ action is extended to $\Sigma_{B}$ by defining

$$
g\left(K_{h}^{R}\right)=K_{g h}^{R} \quad \text { for } 0 \leq R<p ; g, h \in G .
$$

This gives a special $\mathbb{Z} \otimes_{T} G$ action on $\Sigma_{B}$. Define a right resolving, one-to-one a.e. factor map, $\pi$, from $\Sigma_{B} \rightarrow \Sigma_{A}$ by

$$
\begin{aligned}
\pi(I) & =I, & & \text { for } I \in L_{A}, \\
\pi\left(K_{g}^{R}\right) & =g\left(J^{R}\right) & & \text { for all } R \text { and } g .
\end{aligned}
$$

This takes the $\mathbb{Z} \otimes_{T} G$ action on $\Sigma_{B}$ to the one on $\Sigma_{A}$.

Finally, we create, $\Sigma_{\bar{A}}$, an almost conjugate factor of $\Sigma_{B}$. To do this let:

$$
\begin{array}{ll}
L_{\bar{A}}=L_{A} \cup\left\{K_{g}\right\} & g \in G \\
T^{-1} g\left(J_{0}\right) \rightarrow K_{g} & \text { all } g \in G \\
K_{g} \rightarrow K_{T g} & \\
K_{g} \rightarrow L & \text { when } K_{g}^{R} \rightarrow L \text { any } R
\end{array}
$$

This is the image of $\Sigma_{B}$ under the left resolving map $\bar{\pi}(I)=I$ for $I \in L_{A}, \bar{\pi}\left(K_{g}^{R}\right)=K_{g}$. A $G$ action is defined on $\Sigma_{\bar{A}}$ by $g\left(K_{h}\right)=K_{g h}$. The map, $\tilde{\pi}$, takes the special $\mathbb{Z} \otimes_{T} G$ action on $\Sigma_{\mathrm{B}}$ to the one on $\Sigma_{\bar{A}}$. Notice that $K_{e} \rightarrow K_{e}$ so $\Sigma_{\bar{A}}$ has a fixed point. We have the picture

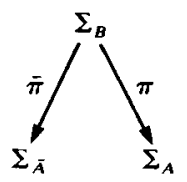


where $\pi$ and $\bar{\pi}$ are one-to-one a.e. factor maps that conjugate the appropriate special $\mathbb{Z} \otimes_{T} G$ actions. $\Sigma_{\bar{A}}$ has a fixed point, and $\bar{\pi}$ is left resolving while $\pi$ is right resolving. We could have made $\bar{\pi}$ right resolving and $\pi$ left resolving by switching the roles of predecessors and successors. This proves the statement at the beginning of this part.

Part (v). Here we observe that the standard tower construction works for $\mathbb{Z} \otimes_{T} G$ actions. Suppose we are given:

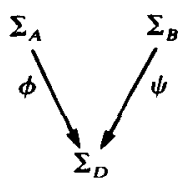

where $\Sigma_{A}, \Sigma_{B}$, and $\Sigma_{D}$ have special $G$ actions, $\phi$ and $\psi$ are factor maps that also commute with these actions. When we do the standard constructions:

$$
L_{C}=\left\{(I, J): I \in L_{A}, J \in L_{B} \text { and } \phi(I)=\psi(J)\right\},
$$

$(I, J) \rightarrow\left(I^{\prime}, J^{\prime}\right)$ if and only if $I \rightarrow I^{\prime}$ in $A$ and $J \rightarrow J^{\prime}$ in $\Sigma_{B}$, we get $\Sigma_{C}$ a cover for $\Sigma_{A}$ and $\Sigma_{B}$.

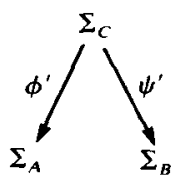

Recall that $\phi^{\prime}$ retains all the characteristics of $\psi$ and $\psi^{\prime}$ all those of $\phi . \Sigma_{C}$ has a $\mathbb{Z} \otimes_{T} G$ action defined on it by $(g(I), g(J))$. This is well-defined and commutes with the maps because

$$
g \circ \phi^{\prime}(I, J)=g(I)=\phi^{\prime}(g(I), g(J)) .
$$

This is all we need. We now put all five parts of this section together for a proof of the theorem stated at the beginning. Given $\Sigma_{A}$ and $\Sigma_{B}$ aperiodic with $\mathbb{Z} \otimes_{T} G$ actions and $\lambda_{A}=\lambda_{B}$, begin (using part (iv)) by finding $\Sigma_{A^{\prime}}, \Sigma_{D}, \Sigma_{B^{\prime}}, \Sigma_{E}$ such that
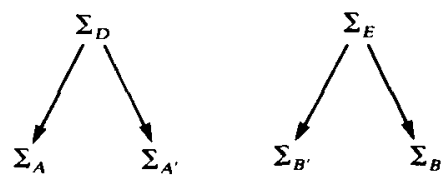

$\Sigma_{A^{\prime}}$ and $\Sigma_{B^{\prime}}$ have fixed points, while all four maps are one-to-one a.e. Next use parts (i), (ii), (iii) to get $\Sigma_{F}$ such that

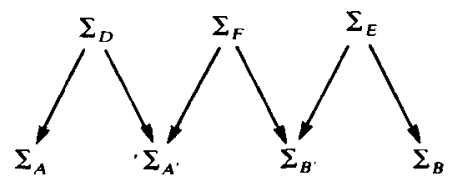

with both maps one-to-one a.e. Finally, use part $(v)$ three times to get: 


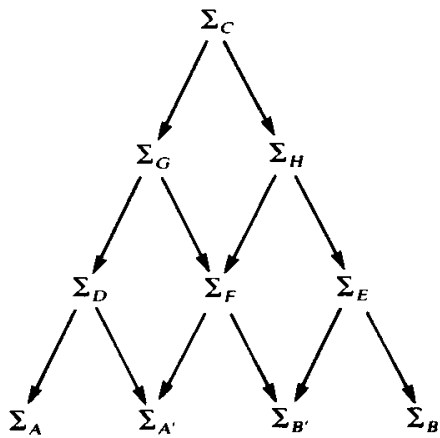

The resulting map $\Sigma_{C} \rightarrow \Sigma_{A}$ is left resolving, one-to-one a.e. and the one $\Sigma_{C} \rightarrow \Sigma_{B}$ is right resolving one-to-one a.e. This completes the proof of the theorem.

\section{The periodic case}

Here we deal with the case where $A$ and $B$ are irreducible but periodic. First, we will give the proof when there is no $G$ acting [1]:

THEOREM 5. Let $\Sigma_{A}$ and $\Sigma_{B}$ be irreducible ssft. They are almost topologically conjugate if and only if they have the same entropy and ergodic period.

Proof. We have the proof when $A$ and $B$ are aperiodic. Suppose $\Sigma_{A}$ and $\Sigma_{B}$ are irreducible with the same entropy and period. Let $\Sigma_{A}^{0}, \ldots, \Sigma_{A}^{p-1}$ and $\Sigma_{B}^{0}, \ldots, \Sigma_{B}^{p-1}$ be the cyclic subsets of $\Sigma_{A}$ and $\Sigma_{B}$, under $\sigma$. This means $\left(\Sigma_{A}^{n}, \sigma^{p}\right)$ and $\left(\Sigma_{B}^{n}, \sigma^{p}\right)$, $0 \leq n<p$, are aperiodic ssft with $p$ times the entropy of $\Sigma_{A}$ or $\Sigma_{B}$. By the previous section there is an aperiodic $\Sigma_{D}$ and one-to-one a.e. factor maps $\theta$ and $\bar{\theta}$ such that

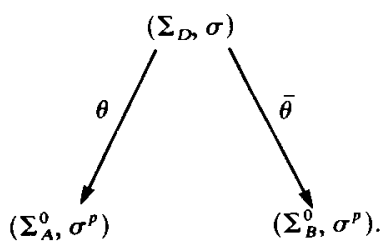

Define $\Sigma_{C}$ an irreducible ssft of period $p$ by:

$$
L_{C}=L_{D} \times\{0, \ldots, p-1\}
$$

and

$$
\begin{aligned}
(I, k) \rightarrow(J, l) & \text { if } I=J \text { and } l=k+1 \leq p-1 \\
& \text { or } I \rightarrow J \text { in } \Sigma_{D} \text { and } l=0, k=p-1 .
\end{aligned}
$$

Notice $\left(\Sigma_{C}^{0}, \sigma^{p}\right)=\left(\Sigma_{D}, \sigma\right)$ so $\theta$ and $\bar{\theta}$ can be considered as maps from $\Sigma_{C}^{0}$ to $\Sigma_{A}^{0}$ and $\Sigma_{B}^{0}$. Now define maps from $\Sigma_{C}$ to $\Sigma_{A}$ and $\Sigma_{B}$ by:

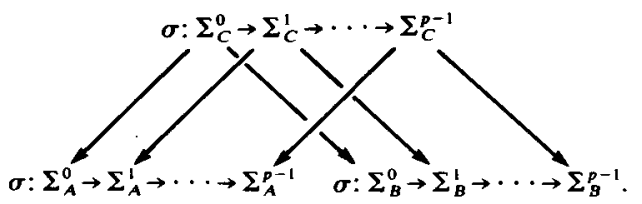


$\pi: \Sigma_{C} \rightarrow \Sigma_{A}$ is defined on each cyclic subset $\pi_{n}: \Sigma_{C}^{n} \rightarrow \Sigma_{A}^{n}$ by $\pi_{n}=\sigma^{n} \circ \theta \circ \sigma^{-n}$ and $\bar{\pi}$ similarly.

Now suppose $\Sigma_{A}$ is an irreducible ssft of period $p>1$ and it has a $\mathbb{Z} \otimes_{T} G$ action. Let $\Sigma_{A}^{0}, \Sigma_{A}^{1}, \ldots, \Sigma_{A}^{p-1}$ be the cyclic decomposition of $\Sigma_{A}\left(\sigma\left(\Sigma_{A}^{\prime}\right)=\Sigma_{A}^{t+1 \bmod p}\right)$. For each $n$ and $t, 0 \leq n, t \leq p-1$ define

$$
H_{t}^{n}=\left\{g \in G: \exists x \in \Sigma_{A}^{\prime} \text { with } g(x) \in \Sigma_{A}^{t+n \bmod p}\right\} .
$$

Then we have:

LEMMA. If the $H_{t}^{n}, 0 \leq t, n<p$ are defined as above,

(i) $g\left(\Sigma_{A}^{t}\right)=\Sigma_{A}^{t+n \bmod p}$, for $g \in H_{t}^{n}, 0 \leq n<p$;

(ii) $H_{t}^{0}$ is a subgroup, $0 \leq t<p$;

(iii) $H_{t}^{1}, \ldots, H_{t}^{p-1}$ are the left cosets for $H_{t}^{0}, 0 \leq t<p$;

(iv) $H_{t}^{n}=T^{i}\left(H_{0}^{n}\right)$;

(v) if $h \in H_{t}^{n}, T^{-n} g \in H_{t}^{m}$ then $g h \in H_{t}^{n+m}$.

Example 5. Define $\Sigma_{A}$ by

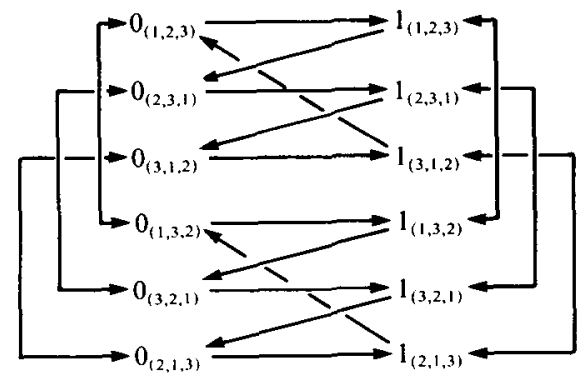

It has period 2 with $L_{A}^{0}=\left\{0_{(1,2,3)}, 0_{(2,3,1)}, 0_{(3,1,2)}, 1_{(1,3,2)}, 1_{(3,2,1)}, 1_{(2,1,3)}\right\}$ and $L_{A}^{1}=\left\{0_{(1,3,2)}\right.$, $\left.0_{(3,2,1)}, 0_{(2,1,3)}, 1_{(1,2,3)}, 1_{(2,3,1)}, 1_{(3,1,2)}\right\}$. Obtain a $\mathbb{Z} \times S(3)$ action by letting $S(3)$ permute the subscripts of the symbols in the natural manner. Then $H_{0}^{0}=H_{1}^{0}=$ $\{(1,2,3),(2,3,1),(3,1,2)\}$ and $H_{0}^{1}=H_{1}^{1}=\{(1,3,2),(3,2,1),(2,1,3)\}$.

Proof. (i) Showing this is true for $t=0$ is sufficient. Take $g \in H_{0}^{n}$, so there is an $I \in L_{A}^{0}$ such that $g(I) \in L_{A}^{n}$. If $J \in L_{A}^{0}$, and $T^{N}=$ identity, then there is an $x$ with $x_{0}=J$ and $x_{l N p}=I$ for some $l$. Then

$$
\sigma^{-l N p} \circ g(x)=T^{-l N p} g \circ \sigma^{-l N p}(x)=g \circ \sigma^{-l N p}(x) \in \Sigma_{A}^{n}
$$

since $\left(\sigma^{-l N p}(x)\right)_{0}=I$; but then $g(x) \in \Sigma_{A}^{n}$ and $g(J) \in L_{A}^{n}$. So $g\left(\Sigma_{A}^{0}\right) \subseteq \Sigma_{A}^{n}$, using the ergodicity of $\sigma^{p}$ on $\Sigma_{A}^{n}$ we have $g\left(\Sigma_{A}^{0}\right)=\Sigma_{A}^{n}$.

(ii) We have $g\left(\Sigma_{A}^{t}\right)=\Sigma_{A}^{t}$ for any $g \in H_{t}^{0}$, so clearly $H_{t}^{0}$ is a subgroup.

(iii) Let $g \in H_{t}^{n}$ then $g^{-1} \in H_{t+n}^{p-n}$ and $g\left(H_{t}^{0}\right) \subseteq H_{t}^{n}$. If $h \in H_{t}^{n}$ then $g^{-1} h \in H_{t}^{0}$ so that $h \in g\left(H_{t}^{0}\right)$ and $H_{t}^{n}=g\left(H_{t}^{0}\right)$.

(iv) Let $T^{t} g \in H_{t}^{n}$, if $x \in \Sigma_{A}^{t}$ then $T^{t} g(x)=T^{t} g \circ \sigma^{t} \circ \sigma^{-t}(x)=\sigma^{t} \circ g \circ \sigma^{-t}(x) \in \Sigma_{A}^{t+n}$. This means $g \circ \sigma^{-t}(x) \in \Sigma_{A}^{n}$ but $\sigma^{-t}(x) \in \Sigma_{A}^{0}$ so $g \in H_{0}^{n}$.

(v) This follows from the above observation. 
Corollary. Suppose we have a $\mathbb{Z} \times G$ action on $\Sigma_{A}$, then we have the following strengthening of the above lemma:

(ii') $H^{0}$ is a normal subgroup;

(iii') $H_{t}^{1}, \ldots, H_{t}^{p-1}$ are left and right cosets for $H^{0}$;

(iv') $H_{0}^{n}=H_{t}^{n}$ for all $n$ and $t$; so we can ignore the subscripts;

(v') if $g \in H^{n}$ and $h \in H^{m}$ then $g h \in H^{n+m}$;

(vi') if $|g|=$ order of $g$, and $g \in H^{n}$ then $n|g|=0 \bmod p$.

Proof. (ii') This follows from (iv').

(iii') This is because $H^{0}$ is normal.

(iv') This follows from (iv) of the lemma.

(v') This follows from (v) of the lemma.

(vi') If $|g|=$ order of $g$, and $g \in H^{n}$ then by (iv') $g^{|g|} \in H^{n|g|}$, but $g^{|g|}=$ id so $n|g|=0 \bmod p$.

These $H_{t}^{n}$ are essential in classifying $\mathbb{Z} \otimes_{T} G$ actions of irreducible but periodic ssft. The corollary tells us that when we have a $\mathbb{Z} \times G$ action we can ignore the subscript. If we have a $\mathbb{Z} \otimes_{T} G$ action, (iv) of the lemma says that the subscripts can be almost, but not entirely ignored. Using these we are led to the main theorem.

THEOREM 6. If there are $\mathbb{Z} \otimes_{T} G$ actions, with $G$ finite, on irreducible ssft $\Sigma_{A}$ and $\Sigma_{B}$ then the two actions are almost topologically conjugate if and only if they have the same topological entropy, the same ergodic period and $H^{0}(A)=H^{0}(B), \ldots, H^{p-1}(A)=$ $H^{p-1}(B)$, (this means for some numbering of the cyclic subsets $H_{0}^{n}(A)=H_{0}^{n}(B)$, $0 \leq n<p)$.

Proof. The necessity of these conditions is clear. We turn to proving the sufficiency. Assume we have fixed $\Sigma_{A}^{0}$ and $\Sigma_{B}^{0}$ so that $H^{n}(A)=H^{n}(B)$, for $0 \leq n<p$. Also assume that we have recoded, and if necessary found almost topological extensions so that the $\mathbb{Z} \otimes_{T} G$ actions are special. The proof breaks down into two cases. The first case is when $H^{1} \neq \varnothing$, and the second is when $H^{1}=\varnothing$. We will deal with them in order.

The first case has $H^{1} \neq \varnothing$ (see example 6). Choose an element $g \in H^{1}$. If we let $g_{n}=\left(T^{n-1} g\right) \cdots(T g) g$, we have that $H_{0}^{n}=g_{n} H_{0}^{0}, 0 \leq n<p$. Now rename the symbols in $L_{A}$ to get $L_{A}^{0} \times\{0, \ldots, p-1\}$ by setting $I \equiv(I, 0)$ and $g_{n}(I) \equiv(I, n)$. This means for any $k \in G, k=g_{n} h$ for some $n$ and $h \in H_{0}^{0}$ and it acts on $L_{A}$ by $k(I, 0)=(h(I), n)$ on $L_{A}^{0}$, and on $L_{A}^{t}$ by $k(I, t)=\left(h^{\prime}(I), m\right)$ where $k g_{n}=g_{m} h^{\prime}$. We are just using $g$ to push the symbols in $L_{A}^{0}$ around through all the symbols in $L_{A}$, and keeping track of the action of $G$. Now we have

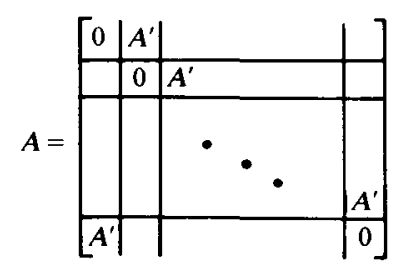

with zeros everywhere else, assuming we have put the symbols in the same order, by the first coordinate, in each cyclic set. $A^{\prime}$ is aperiodic; as it stands it defines 
transitions from $(I, n) \rightarrow(J, n+1) \bmod p$. Define a new ssft $\Sigma_{A^{\prime}}$ by $L_{A^{\prime}}=L_{A}^{0}$ and $(I, 0) \rightarrow(J, 0)$ when $(I, n) \rightarrow(J, n+1)$. Define a special $\mathbb{Z} \otimes_{T^{\prime}} H^{0}$ action on $\Sigma_{A^{\prime}}$ by letting $h \in H^{0}$ act on $(I, 0)$ as it did in $\Sigma_{A}$. Define $T^{\prime}: H^{0} \rightarrow H^{0}$ by saying

$$
T^{\prime} h=g^{-1} \circ T h \circ g \text {. }
$$

Repeat this process on $\Sigma_{B}$, using the same element $g$, to get an aperiodic $\Sigma_{B^{\prime}}$ with a $\mathbb{Z} \otimes_{T^{\prime}} H^{0}$ action on it. Apply the results of the previous section to get $\Sigma_{D}$ with a $\mathbb{Z} \otimes_{T^{\prime}} H^{0}$ action that is an almost conjugate extension of $\Sigma_{A^{\prime}}$ and $\Sigma_{B^{\prime}}$. Now define $\Sigma_{C}$ by

$$
L_{C}=L_{D} \times\{0, \ldots, p-1\}
$$

and

$$
(I, n) \rightarrow(J, n+1) \quad \text { if } I \rightarrow J \text { in } \Sigma_{D}
$$

Define a $\mathbb{Z} \otimes{ }_{T} G$ action on this by saying, for $k \in G, k=g_{n} h, k(I, 0)=(H(I), n)$ on $L_{C}^{0}$, and $k(I, t)=\left(h^{\prime}(I), m\right)$ where $k g_{t}=g_{m} h^{\prime}$ in general. We define $\pi: \Sigma_{C} \rightarrow \Sigma_{A}$ by $\pi(I, n)=(\theta(I), n)$ and observe that this takes the $\mathbb{Z} \otimes{ }_{T} G$ action on $\Sigma_{C}$ to the one on $\Sigma_{A}$. Then define $\bar{\pi}$ in the same way.

The final case is when $H^{1}=\varnothing$. To treat this we let $p^{\prime}=\operatorname{gcd}\left\{r: H^{r} \neq \varnothing\right\}$. Observe that

$$
\left\{n p^{\prime}(\bmod p): n=1,2, \ldots\right\}=\left\{r: H^{r} \neq \varnothing\right\}
$$

and so $p=l p^{\prime}$ for some $l$. Consider $\sigma^{p^{\prime}}$ on $\Sigma_{A}$; it will not be irreducible. There will be $p^{\prime}$ irreducible components, each of period $l$. Consider the subshift of finite type, $\Sigma_{E}$, that is made up of the orbit of $\Sigma_{A}^{0}$ under $\sigma^{p^{\prime}}$, with $\sigma^{p^{\prime}}$ acting on it. This has a natural $\mathbb{Z} \otimes_{S} G$ action on it where $S=T^{p^{\prime}}$ and $G$ is the original group acting in the original manner. Define $\Sigma_{F}$ in the same manner beginning with $\Sigma_{B}$. This also has a natural $\mathbb{Z} \otimes_{S} G$ action on it.

Consider $\Sigma_{E}$ and $\Sigma_{F}$ with their $\mathbb{Z} \otimes_{S} G$ actions. This is the case we just dealt with; $H^{\prime}(E)=H^{p^{\prime}}(A) \neq \varnothing$. Build $\Sigma_{D}$ with a $\mathbb{Z} \otimes_{S} G$ action that is an almost conjugate extension of the $\mathbb{Z} \otimes_{S} G$ actions on $\Sigma_{E}$ and $\Sigma_{F}$.

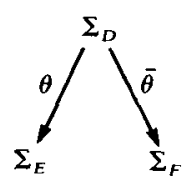

Now define a new ssft $\Sigma_{C}$ of period $p$ by

$$
L_{C}=L_{D}^{0} \cup L_{D}^{1} \cup \cdots \cup L_{D}^{l-1} \times\left\{0, \ldots, p^{\prime}-1\right\}
$$

and

$$
\begin{array}{ll}
(I, n) \rightarrow(I, n-1) & \text { for } 0 \leq n \leq p^{\prime}-2 \\
\left(I, p^{\prime}-1\right) \rightarrow(J, 0) & \text { where } I \rightarrow J \text { in } \Sigma_{D} .
\end{array}
$$

This has a $\mathbb{Z} \otimes_{T} G$ action defined on it by $g(I, n)=(g(I), n)$. Finally define maps $\pi: \Sigma_{C} \rightarrow \Sigma_{A}$ and $\bar{\pi}: \Sigma_{C} \rightarrow \Sigma_{B}$ as at the beginning of this section. They are defined on each cyclic subset $\pi_{n}: \Sigma_{C}^{n} \rightarrow \Sigma_{A}^{n}$ by $\pi_{n}=\sigma^{m} \circ \theta \circ \sigma^{-m}$ where $m$ is $n$ after reduction $\bmod p^{\prime}$. These maps take the $\mathbb{Z} \otimes_{T} G$ action on $\Sigma_{C}$ to the ones on $\Sigma_{A}$ and $\Sigma_{B}$. 
The condition that $H^{0}(A)=H^{0}(B), \ldots, H^{p-1}(A)=H^{p-1}(B)$ is just a different statement of the same condition obtained by D. Rudolph [7] in the measure-theoretic case. Example 6. Let $\Sigma_{A}$ with its $\mathbb{Z} \times S(3)$ action be as in example 5. Choose $g=(1,3,2) \in$ $H^{1}$. Then the $\Sigma_{A^{\prime}}$ with its $\mathbb{Z} \otimes_{T^{\prime}} H^{0}$ action that results from the construction in the previous proof is the one described in example 2 .

\section{Sofic systems}

The following theorems can be proved using the ideas contained in the previous sections and a few facts about sofic systems. We include them for completeness, but leave out the proofs.

THEOREM 7. If $S$ is any positive entropy sofic system, $G$ is any finite group, and $T$ is any automorphism of $G$ then there is $a \mathbb{Z} \otimes_{T} G$ action on $S$.

THEOREM 8. If $S$ is an irreducible sofic system with a $\mathbb{Z} \otimes_{T} G$ action then there is a ssft equipped with a $\mathbb{Z} \otimes_{T} G$ action that is an almost conjugate extension of the one on $S$.

COROLlary. If $S$ and $S^{\prime}$ are two irreducible sofic systems that have $\mathbb{Z} \otimes_{T} G$ actions, then these actions are almost topologically conjugate if and only if they have the same topological entropy, the same ergodic period, and $H^{0}(S)=H^{0}\left(S^{\prime}\right), \ldots, H^{p-1}(S)=$ $H^{p-1}\left(S^{\prime}\right)$.

\section{REFERENCES}

[1] R. L. Adler \& B. Marcus. Topological entropy and equivalence of dynamical systems. Mem. Amer. Math. Soc. 219 (1979).

[2] R. L. Gray. Generalizing period and topological entropy to transitive non-wandering systems. Masters thesis, Univ. of NC, Chapel Hill, 1978.

[3] G. A. Hedlund. Endomorphisms and automorphisms of the shift dynamical system. Math. Systems Theory 3 (1969), 320-375.

[4] W. Krieger. On the periodic points of topological Markov chains. Math. Z. 169 (1979), 99-104.

[5] W. Parry. A finitary classification of topological Markov chains and sofic systems. Bull. London Math. Soc. 9 (1977), 86-92.

[6] D. J. Rudolph. Counting the relatively finite factors of a Bernoulli shift. Israel J. Math. 30, No. 3 (1978), 255-263.

[7] D. J. Rudolph. An isomorphism theory for Bernoulli $Z$-skew compact group actions. Advances in Math. 47, No. 3 (1983), 241-257. 